Arbeitgeber muss Ex-Mitarbeiter aus dem Web löschen

Arztpraxen, Kliniken und andere Arbeitgeber müssen Daten ausgeschiedener Arbeitnehmer von ihrer Homepage löschen. So entschied das Hessische Landesarbeitsgericht (LAG) die Klage einer Anwältin (19 SaGa 1480/11). Ihr Eintritt in eine Kanzlei wurde mit Foto auf der Homepage sowie in einem NewsBlog bekanntgegeben. Als sie nach drei Monaten wieder ausschied, löschte die Kanzlei die Daten von der Homepage, nicht aber aus dem Blog. Das LAG entschied, dass ausgeschiedene Arbeitnehmer die Löschung ihrer Daten aus allen Internetauftritten ihrer Ex-Arbeitgeber verlangen können.

Martin Wortmann

\section{Rückstellung nur mit}

Regressbescheid

Ärzte dürfen erst dann steuerlich wirksame Rückstellungen für Regressforderungen bilden, wenn die Prüfgremien einen Regressbescheid erlassen haben. Das hat das Finanzgericht Bremen entschieden (1 K 32/10 [5]). Stützen sich die Rückstellungen nur auf ein eingeleitetes Prüfverfahren, sind sie steuerlich unwirksam. Im verhandelten Fall wurde gegen eine Gemeinschaftspraxis ein Prüfverfahren eingeleitet, weil sie die maßgeblichen Richtgrößen für die Verordnung von Arznei- und Heilmitteln erheblich überschritten hatte. Die Ärzte hatten in ihren Bilanzen deshalb gewinnmindernde Rückstellungen wegen der befürchteten Festsetzung von Regressen gebildet. Zu Unrecht, so das Gericht. Rebekka Höhl

\section{Vermittlungsprovision unzulässig}

Für die Vermittlung von Patienten an eine Klinik dürfen Ärzte generell keine Provisionen nehmen. Das gilt selbst für Privatpatienten aus dem Ausland, heißt es in einem Urteil des Landgerichts Kiel (8 O 28/11). Es verwarf damit den Provisionsvertrag zwischen einem Arzt und dem Uniklinikum Schleswig-Holstein als sittenwidrig. Der Arzt spricht fließend Arabisch und verfügt über gute Kontakte in den arabischen Raum. Der Arzt sollte laut Vertrag ausländische Privatpatienten vermitteln und sie anschließend während des Klinikaufenthalts begleiten und dolmetschen.

\section{Praxischefs müssen seit April mehr Gehalt zahlen}

\author{
Ein Plus von 2,9\% für Medizinische Fachangestellte (MFA) und von rund \\ $8 \%$ für Auszubildende - so sieht der neue MFA-Tarif aus.
}

S eit April sind die Tarifgehälter für Medizinische Fachangestellte (MFA) gestiegen. Anders als bei der Tarifrunde 2011, als das Plus für Berufseinsteiger höher lag als das für erfahrene Kräfte, gibt es dieses Mal für alle MFA ein und dasselbe Lohnplus, nämlich 2,9\% auf das bisherige Tarifgehalt. Darauf haben sich der Verband der medizinischen Fachberufe (VmF) und die Arbeitsgemeinschaft zur Regelung der Arbeitsbedingungen von Medizinischen Fachangestellten/Arzthelferinnen (AAA) geeinigt.

\section{Auszubildende bekommen noch mehr}

Ein dickeres Gehaltsplus gibt es für die Auszubildenden: Sie erhalten ein Plus zwischen $7 \%$ und $8 \%$ : Das Gehalt für das erste Ausbildungsjahr steigt von 561 auf nun 610 Euro, für das zweite Ausbildas dritte Ausbildungsjahr von 646 auf 700 Euro.

Sein Ziel, für jede MFA mindestens einen Stundenlohn von 10 Euro auszuhandeln, habe der VmF mit dem neuen Abschluss leider noch nicht erreicht, sagt Margret Urban, zuständig für das Ressort Tarifpolitik beim Verband. Man liege bei den Berufseinsteigern durch den neuen Tarif bei einem Stundenlohn von 9,21 Euro. „Wir hätten schon gerne eine drei vor dem Komma gehabt", so Urban. Die Vorsitzende der AAA, Dr. Cornelia Goesmann, hingegen sieht in den 2,9\% die Grenze dessen, was für die Ärzteschaft möglich war.

RebekkaHöhl dungsjahr von 602 auf 650 Euro und für

Abrechungstipp

\section{Nebenleistungen nicht verschenken}

Auch vermeintlich kleine Tätigkeiten „im Vorbeigehen“ sollte man korrekt abrechnen. Immer wieder gibt es beispielsweise Unstimmigkeiten darüber, welche Leistungen in der Abrechnung nach GOÄ neben den Gelenkpunktionen (Nummern 300-302) abrechenbar sind. Zunächst bleibt festzuhalten, dass es für die Punktion von Ellenbogen-, Knie- oder Wirbelgelenken eine eigenständige Abrechnungsposition gibt (Nr. 301/160 Punkte), ebenso für die Punktion des Schulter- oder Hüftgelenks (Nr. 302/250 Punkte). Für alle anderen Gelenkpunktionen bleibt die Nr. 300 mit 120 Punkten. Allen drei Positionen gemeinsam ist der Ausschluss der gleichzeitigen Positionen für Arthroskopien (Nrn. 2189, 2190, 2191, 2192, 2193, 2195, 2196) sowie von Kontrastuntersuchungen einschließlich Punktion (Nrn. 5050, 5060, 5070).

Neben den Punktionen sind weiterhin abrechenbar die Infiltrationsanästhesie kleiner Bezirke (Nr. 490/61 Punkte) zur schmerzärmeren Punktion, die intraartikuläre Infiltration (Nr. 255/95 Punkte) sowie das Anlegen eines Kompressionsverbands nach der Punktion. Hierbei ist zu beachten, dass der einfache elastische Verband zur Abdeckung der Punktionsstelle ein Verband nach Nr. 200 und damit laut Präambel nicht neben Punktionen abrechenbar ist. Muss aber beispielsweise ein Schaumstoffkompressionsverband angelegt werden, um das Nachlaufen eines Ergusses zu verhindern, ist dieser durchaus mit der Nr. 204 (95 Punkte) abrechenbar. Bei traumatischen Ergüssen, etwa am Knie, kann auch ein anschließender Schienenverband fällig werden, wenn beispielsweise der Verdacht auf eine Innenbandverletzung besteht. Gleich zu Beginn würde man dann auch eine subkutane Thromboseprophylaxe mit einem niedermolekularen Heparin ansetzen (Nr. 252/40 Punkte).

Neben den Abrechnungsziffern sind zusätzliche Sachkosten für verwandte Arbeitsmittel abzurechnen. Hier kann man hilfsweise auf die in der UV-GOÄ aufgelisteten besonderen Kosten zurückgreifen, die zu einzelnen Ziffern festgesetzt sind, ebenso die Kosten für verbrauchte Ampullen. 